\title{
COMMENT
}

\section{Impact of the Innovative Medicines Initiative on vaccine development}

\author{
Philippe Denoel ${ }^{1 *}$, Patricia Londoño-Hayes ${ }^{2}$, Magda Chlebus ${ }^{3}$ and \\ Magdalena Rodriguez de Azero ${ }^{4}$
}

Since its launch a decade ago, the European Innovative Medicines Initiative (IMI), a leading

public-private partnership in life sciences, has catalysed scientific breakthroughs and the

development of strategies to address complex challenges in various biomedical fields.

Here, we discuss the progress of IMI projects related to vaccine research and development.

In 2008, the European Commission and the European Federation of Pharmaceutical Industries and Associations (EFPIA) founded the Innovative Medicines Initiative (IMI) to address bottlenecks in pharmaceutical R\&D through collaborative research and open innovation. IMI has since fostered many public-private partnerships (PPPs) between regulators, industry, academia, patient groups and small/medium-sized enterprises (SMEs).

In IMI's first phase ('IMI1'), 40\% of its $€ 2$ billion budget was invested in infectious disease research, and so vaccines were implicitly within scope. However, less than $5 \%$ of this budget was allocated to vaccine research, and it was not until 2012 that the first vaccine-focused project, 'Biomarkers for Enhanced Vaccine Safety' (BIOVACSAFE), was initiated. The strategic research agenda (SRA) for the next phase ('IMI2', from 2014-2020, with a $€ 3.3$ billion budget) specifically included vaccine development as a priority. This intensified vaccine focus has led to the launch of fourteen vaccine-focused consortia so far (Supplementary Figure 1). Relative to the non-vaccine projects, these projects have received similar in-kind contributions from the EFPIA, while typically having fewer EFPIA partners. This demonstrates substantial support from the vaccine sector, which is only a segment of the pharma industry in Europe. Although still consuming a modest proportion of the budget ( $10 \%$ for IMI2), these projects have produced major contributions to vaccine $\mathrm{R} \& \mathrm{D}$ innovation in Europe and beyond (Supplementary Table 2). In this article, we discuss the key successes and their impact.

${ }^{2}$ Sanofi Pasteur,

Marcy-l'Étoile, France.

${ }^{3}$ European Federation of

Pharmaceutical Industries and Associations (EFPIA),

Brussels, Belgium.

${ }^{4}$ Vaccines Europe/EFPIA,

Brussels, Belgium.

*e-mail:

philippe.denoel@gsk.com

doi:10.1038/nrd. 2018.72

Published online 1 Jun 2018 resolve the scientific and technological bottlenecks in early-phase R\&D cannot be delivered by manufacturers on an individual basis. By building a critical mass of research efforts by private and public stakeholders, IMI consortia have facilitated the translation of basic immunology insights into vaccine development efforts - for instance, by using systems biology approaches - while enabling the burden and risks to be shared.

For example, a landmark in the quest for safety biomarkers by the BIOVACSAFE consortium was the identification of early immune patterns that predict persistent immunity against influenza, including $\mathrm{T}$ follicular helper cell responses ${ }^{1}$. Similarly, the efforts made by the VSV-EBOVAC consortium, aiming to characterize recombinant vesicular stomatitis virus (rVSV)-Ebola vaccine safety and immunity, led to the identification of a unique early response plasma signature of adverse events and vaccine-induced immune responses ${ }^{2}$. As this signature proved consistent across different settings and genetic backgrounds, it may be suitable for other vaccine evaluations. Furthermore, biomarkers of durable vaccine-generated immunity are being explored by the PERtussIS COrrelates of Protection Europe (PERISCOPE) consortium ${ }^{3}$, IMI's first project to receive funding from the Bill and Melinda Gates Foundation.

\section{Advancing clinical evaluation via collaboration}

These achievements have been facilitated by the data generated in the clinical vaccine or epidemiology studies launched by these consortia, and the associated peer-reviewed publications have increased the scientific knowledge base. The wealth of data generated in the 14 exploratory BIOVACSAFE trials reflects the strength of collaborative research. Indeed, in an analysis covering 28 of these publications, their overall citation impact was found to be three times the world average (Supplementary Table 2). Additional examples include the recently launched 'BERT' trial, the first of nine cross-border PERISCOPE studies assessing pertussis vaccine-induced 
immunity, and four respiratory syncytial virus (RSV) epidemiology studies launched by the REspiratory Syncytial virus Consortium in EUrope (RESCEU) consortium. Additionally, multiple phase I and II Ebola vaccine trials have been initiated in the EBOVAC1 and 2 consortia as part of the EBOLA+ program, founded in response to the outbreak in Western Africa in 2014.

\section{Addressing knowledge fragmentation}

In late-stage development, IMI consortia have fostered an open pre-licensure dialogue between manufacturers and public parties, promoting the sharing of expertise, technologies and data and optimized use of trial data, models and assays. This is exemplified by the RESCEU project, in which reopening of databases from completed clinical trials by industry partners has enabled a major data-mining exercise supporting the determination of RSV incidence in Europe's elderly. At a global level, the PERISCOPE project has enabled the most widely accepted preclinical pertussis model to be shared between US and French national public health organizations, allowing its future use by manufacturers and the research community.

Another landmark was the first-ever collaboration between the vaccine industry and public partners accomplished by the inFLUenza vaccine Correlates Of Protection (FLUCOP) consortium, which has advanced development of promising assays that offer simplified procedures and the potential of future standardization ${ }^{4}$. This helps tackle the high intra- and inter-laboratory variabilities in routine assays. Indeed, for both the haemagglutination inhibition and virus neutralization assays, used by public and private sectors for influenza vaccine registration and clinical trials, such variabilities can amount to up to 6-7-fold differences in outcomes, posing a persistent conundrum in the standardized interpretation of vaccine immunogenicity and efficacy data. The collective efforts of FLUCOP and the recently initiated DRIVE consortium, which are aiming to further improve European partnerships to conduct influenza vaccine effectiveness studies, are expected to substantially enhance the timely evaluation of current and future vaccines against this disease.

\section{Fostering open communication}

Last, IMI projects have offered a neutral environment in which the sharing of post-licensure monitoring data and discussions on vaccine coverage, risks and benefits between stakeholders have flourished, thus promoting alignment between industrial, public health and regulatory parties. The need for such a platform became apparent in the aftermath of the $2009 \mathrm{H} 1 \mathrm{~N} 1$ pandemic, and prompted the launch of the Accelerated Development of VAccine benefit-risk Collaboration in Europe (ADVANCE) consortium. By critically reviewing current guidelines, this PPP has produced four white papers describing a proposed code of conduct for collaborative vaccine benefit-risk monitoring studies, and the proof of concept has recently been obtained ${ }^{5}$ (Supplementary Table 2). This paves the way for future assessments, and may aid the most recent IMI vaccine call in the context of the European efforts supporting healthy ageing.
This consortium will use a holistic approach that translates basic immunology, epidemiology and health economics data into recommendations for a comprehensive vaccination programme for the elderly.

\section{Conclusions and looking forward}

By synergizing the strengths of the stakeholders, IMI consortia have contributed substantially to innovation in vaccine $R \& D$, and it is expected that many of these collaborations will progress into durable scientific networks. Industry's strong commitment to the PPP framework has been instrumental in shaping this success. In addition to providing half of IMI2's vaccine budget (equivalent to €170 million; typically contributed 'in kind'; Supplementary Box 3), EFPIA companies have been active at the operational project level and in setting the SRA, as evidenced by the high proportion of resourceintensive projects with a strong industry involvement.

Despite the different drivers for academia and industry, and barriers facing cross-pharma collaborations, IMI consortia have created a considerable level of engagement and trust among its multiple participants ( 20 in a typical vaccine project), which was aided by the opportunity for all stakeholders to contribute to the SRA. In earlier consortia such as BIOVACSAFE and ADVANCE, which cooperated strictly within the precompetitive space, IMI's knowledge dissemination policy allowed for unprecedented levels of sharing of data and expertise. Nevertheless, tighter collaboration in competitive areas was recognized as a key ingredient for increased scientific output, and the launch of IMI2 signalled a shift towards a more collaborative way of working towards shared goals in more project-specific areas, thereby approaching the competitive boundaries normally present in antigen discovery efforts. FLUCOP and PERISCOPE, pursuing joint identification of correlates of protection, are representative of this approach.

Finally, future challenges include expanding the currently mainly disease-driven projects towards development of platforms that also encompass technologyoriented research - for example, in vaccine adjuvants or other innovative technologies - as well as attracting more associated partners, non-EU partners and consolidating the SME stakeholders' engagement in the projects.

1. Spensieri, F et at. Early rise of blood T follicular helper cell subsets and baseline immunity as predictors of persisting late functional antibody responses to vaccination in humans. PLOS ONE 11 e0157066 (2016).

2. Huttner, A. et al. A dose-dependent plasma signature of the safety and immunogenicity of the rVSV-Ebola vaccine in Europe and Africa. Sci. Transl Med. 9, eaaj1701 (2017).

3. de Graaf, $\mathrm{H}$. et al. Investigating Bordetella pertussis colonisation and immunity: protocol for an inpatient controlled human infection model. BMJ Open 7 , e018594 (2017).

4. de Vries, R. D. et al. Influenza virus-specific antibody dependent cellular cytoxicity induced by vaccination or natural infection Vaccine 35, 238-247 (2017).

5. Kurz, X et al. The ADVANCE Code of Conduct for collaborative vaccine studies. Vaccine 35, 1844-1855 (2017).

Acknowledgements

The authors are grateful to L. Cochard (SCINNOV) for helpful discussions, and E. Oe and U. Krause (both GSK) for scientific writing services and publication management, respectively.

Competing interests

The authors declare competing interests: see Web version for details.

Supplementary information

Supplementary information is available for this paper at

http://www.nature.com/articles/nrd.2018.72 